\title{
Chaotic properties of a time-modulated barrier
}

\author{
E. D. Leonel and P. V. E. McClintock \\ Department of Physics, Lancaster University, Lancaster LA1 4YB, United Kingdom
}

(Received 8 January 2004; published 26 July 2004)

\begin{abstract}
Some chaotic properties of a classical particle interacting with a time-modulated barrier are studied. The dynamics of this problem is obtained by use of a two-dimensional nonlinear area-preserving map. The chaotic low energy region is characterized in terms of Lyapunov exponents. The time that the particle stays trapped in the well is such that the distributions of successive reflections, and of the corresponding successive reflection times, obey power laws with the same exponent. Using time series analysis, we show that the chaotic sea exhibits an interesting scaling property over a large range of control parameters. Our results indicate that the particle experiences unlimited energy growth when the barrier behaves randomly.
\end{abstract}

DOI: 10.1103/PhysRevE.70.016214

PACS number(s): 05.45.Pq

\section{INTRODUCTION}

The problem of a particle interacting with a timemodulated barrier has received close attention in recent years. In the quantum case, a question of particular interest relates to the time involved in the tunneling process. Although this subject is quite well understood [1], some controversial results were pointed out in [2]. Since then, different approaches have been used and much attention has been concentrated on both the quantum [3-7] and classical versions of the problem $[8,9]$. In the quantum case, several important results were obtained, including the frequency dependence of the tunneling time [2], the transmission probability spectrum in a driven triple diode in the presence of a periodic external field [3], photon-assisted tunneling through a GaAs $/ \mathrm{Al}_{x} \mathrm{Ga}_{1-x} \mathrm{As}$ quantum dot induced by an external microwave field [4], sequential tunneling in a super-lattice induced by an intense electric field [5], electron transmission resonance above a quantum well due to dissipation [6] and the probability of dissipative tunneling in Josephson junction circuits [7]. The corresponding classical problem was studied by Mateos and José [8], and Mateos [9]. In these latter papers they showed that the dynamics of a particle inside an infinite potential box that contains a periodically oscillating barrier presents a rich hierarchy of behaviors. With an appropriate adjustment of control parameters and initial conditions, periodic, quasi-periodic and chaotic motion may all be observed. The authors studied the dynamics using an areapreserving map in energy-time variables. The most significant finding presented in those papers was that the traversal time, i.e., the length of time taken by the particle to traverse the oscillating barrier, obeys a power law distribution of exponent -3 . This distribution provides clear evidence of chaotic behavior, and the authors suggest that the exponent may be indicative of scale invariance. It is also interesting to study the problem of a classical particle interacting with a static or time-dependent multi-well potential in the presence of noise. Recent investigations include population changes in the presence of an external field [10], the escape flux from a multi-well metastable potential preceding the formation of quasi-equilibrium [11], activation over a randomly fluctuat- ing barrier [12,13], and diffusion across a randomly fluctuating barrier [14]. The notion of the time dependent potential may be also extended to encompass the class of problems known as billiards [15-18].

In this paper we revisit the problem of a classical particle interacting with an infinitely deep potential well containing a time-dependent potential barrier, seeking to understand some chaotic properties of this system. First, we consider the case of a periodically varying barrier and then, second, we consider one that is stochastically perturbed. For the periodic case, we derive a two-dimensional map that describes the dynamics of the particle. As we will see, the phase space is extremely complex and shows a set of invariant spanning curves in the high energy regime, and KAM islands surrounded by a chaotic sea at low energies. For intermediate values of energy it is possible to observe some chaotic regions limited by two different invariant spanning curves. We concentrate on the low energy region located below the first invariant spanning curve, and we evaluate the Lyapunov exponents for the chaotic region using a large range of control parameters. Using roughening concepts, we obtain a scaling function as well as the critical exponents for chaotic time series located in the chaotic sea. The scaling function is supported by a very good collapse of the roughness data for different control parameters onto a single curve. We also study the distribution of successive reflections in terms of both reflection number and reflection time for a particle trapped in a well created by the oscillating potential. For a stochastic perturbation, we will show that the particle may have unlimited energy growth once the stochastic perturbation destroy the invariant spanning curves and KAM islands.

The paper is organized as follows. In Sec. II, we describe how the map is constructed. We discuss how to obtain successive reflections and how to evaluate their corresponding times for a particle trapped in the well created by the oscillating barrier. We also discuss briefly the method used to obtain the Lyapunov exponents. The numerical results and scaling properties for the periodic case are discussed in Sec. III. The stochastic version for the problem is developed in Sec. IV. In Sec. V we summarize the main results and present concluding remarks. 


\section{NUMERICAL TECHNIQUES: THE MODEL AND LYAPUNOV EXPONENT CALCULATIONS}

\section{A. The model with periodic oscillations}

We consider a particle inside an infinite potential box that contains an oscillating barrier. It consists basically of a onedimensional system that may be described by a timedependent Hamiltonian. Let us define the Hamiltonian for this system as $H(x, p, t)=p^{2} / 2 m+V(x, t)$, where $V(x, t)$ is given by $V(x, t)=V_{0}(x)+V_{1}(x, t)$. The potential $V_{0}(x)$ is defined as

$$
V_{0}(x)= \begin{cases}0, & \text { if } 0<x<l \text { and } l+b<x<l+b+L \\ V_{0}, & \text { if } l \leqslant x \leqslant l+b \\ \infty, & \text { if } x \leqslant 0 \text { and } x \geqslant l+b+L\end{cases}
$$

and $V_{1}(x, t)$ is written as

$$
V_{1}(x, t)=\left\{\begin{array}{c}
0, \text { if } x<l \text { and } x>l+b \\
V_{1} \sin (t), \text { if } l \leqslant x \leqslant l+b
\end{array}\right.
$$

where $V_{0}$ is constant and gives us the height of the static potential barrier. $V_{1}$ is the amplitude of oscillation in the region $l \leqslant x \leqslant l+b$. We analyze the case $V_{1}<V_{0}$. The parameters $l, b$ and $L$ are, respectively, the widths of well I, of the barrier and of well II as shown in Fig. 1. The dynamics of a particle interacting with an oscillating potential barrier may be described by a two-dimensional map $T$ that gives the total energy of the particle and the time at which the particle collides with an infinite potential wall located at $x=0$, i.e., $\left(E_{n+1}, t_{n+1}\right)=T\left(E_{n}, t_{n}\right)$. To derive the map $T$, we will follow a similar procedure to that used in Ref. [9]. Suppose that the particle start from $x=0$ at time $t_{n}$ with initial energy $E_{n}$ $=K_{n}=m v_{n}^{2} / 2>V_{0}-V_{1}$. If $E_{n}<V_{0}-V_{1}$, the particle will never escape from well I. Starting from $x=0$, the length of time it takes to reach the left-hand side of the barrier at $x=l$ is $t_{n}^{\prime}$ $=l / v_{n}$, where $v_{n}=\sqrt{2 K_{n} / m}$. In this situation, two different kinds of behavior may occur, depending on the energy of the particle. If $E_{n}>V_{0}+V_{1} \sin \left(t_{n}+t_{n}^{\prime}\right)$, then the particle is transmitted past the potential and we will use an application that we call $T_{B}$; otherwise, it is reflected with the same initial energy to the left side of the barrier and we use $T_{A}$. Let us first derive $T_{A}$. When the particle is reflected to the left side with the same initial energy and has a kick from the wall located at $x=0$, the new energy is $E_{n+1}=E_{n}$ and the new time is $t_{n+1}=t_{n}+2 l / v_{n}$. After that, the particle starts again from $x$ $=0$ and travels to the right with the same velocity until it arrives at the left side of the potential barrier $(x=l)$. If $E_{n+1}<V_{0}+V_{1} \sin \left(t_{n+1}+t_{n+1}^{\prime}\right)$, the particle will be reflected again to the left side, and so on, until it is able to traverse the barrier. In this case, the application $T_{A}$ at the $(n+1)$ th kick can be written as

$$
T_{A}:\left\{\begin{array}{l}
E_{n+1}=E_{n}, \\
t_{n+1}=t_{n}+2 l / \sqrt{2 E_{n+1} / m} .
\end{array}\right.
$$

Next, we derive $T_{B}$. If the particle has total energy $E_{n}>V_{0}$ $+V_{1} \sin \left(t_{n}+t_{n}^{\prime}\right)$ when it arrive at $x=l$, then it will be transmitted. The particle experiences an abrupt change in its kinetic energy, and the new expression is $K_{n}^{\prime}=E_{n}-\left[V_{0}+V_{1} \sin \left(t_{n}\right.\right.$

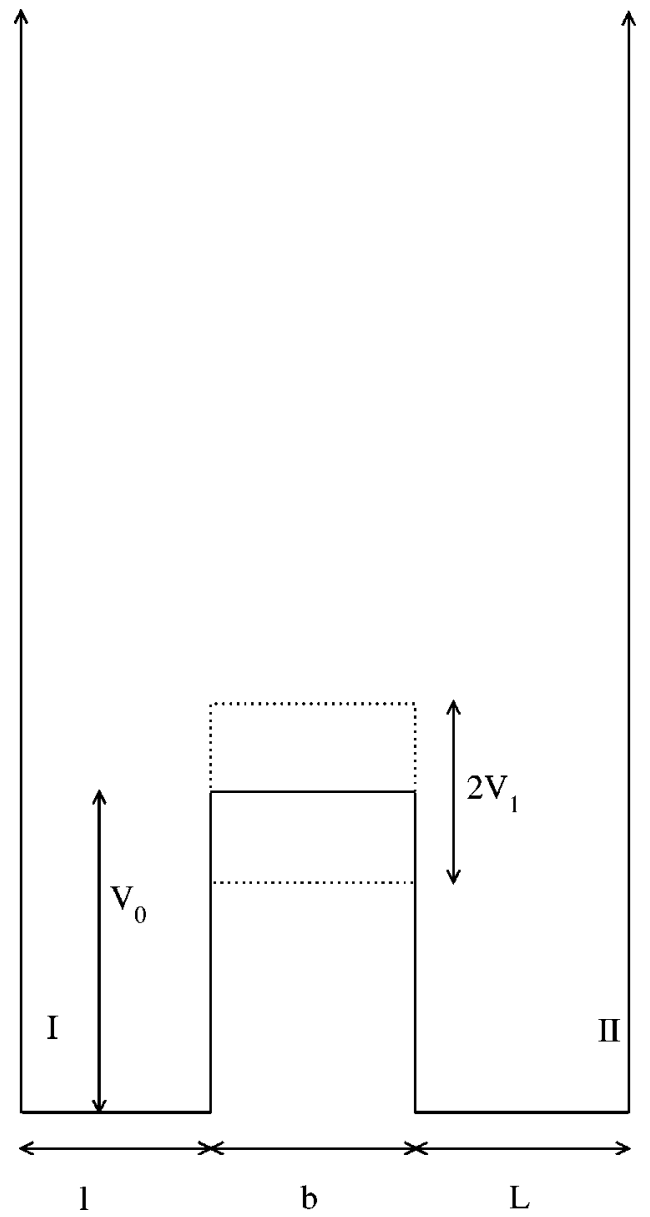

FIG. 1. A sketch of the potential $V(x, t)$ for the periodic case; the zeroes of $x$ and $V$ are in the bottom left-hand corner.

$\left.\left.+t_{n}^{\prime}\right)\right]$. After traveling a distance $b$ at the constant velocity $v_{n}^{\prime}=\sqrt{2 K_{n}^{\prime} / m}$ the particle arrives at $x=l+b$ after a time $t_{n}^{\prime \prime}$ $=b / v_{n}^{\prime}$. Again, the particle changes its kinetic energy abruptly to a new value $E_{n}^{\prime \prime}=K_{n}^{\prime \prime}=K_{n}^{\prime}+V_{0}+V_{1} \sin \left(t_{n}+t_{n}^{\prime}+t_{n}^{\prime \prime}\right)$. It then travels within well II at a constant velocity $v_{n}^{\prime \prime}$ $=\sqrt{2 K_{n}^{\prime \prime} / m}$. After a time $t_{n}^{\prime \prime \prime}=L / v_{n}^{\prime \prime}$ the particle will be reflected with the same kinetic energy towards the left-hand side of the potential after suffering an elastic collision with the wall of the potential located at $x=l+b+L$. When the particle arrives at the right-hand side of the barrier $(x=l+b)$, two possibilities arise: (a) it will be transmitted if $E_{n}^{\prime \prime}>V_{0}$ $+V_{1} \sin \left(t_{n}+t_{n}^{\prime}+t_{n}^{\prime \prime}+2 t_{n}^{\prime \prime \prime}\right)$; or otherwise, (b) the particle will be reflected with the same velocity back towards the right hand side of the well II until it satisfies the condition $E_{n}^{\prime \prime}>V_{0}$ $+V_{1} \sin \left(t_{n}+t_{n}^{\prime}+t_{n}^{\prime \prime}+2 i t_{n}^{\prime \prime \prime}\right)$. Here, $i$ is the smallest integer for which the last equation is true.

We emphasize that for $i>1$, the number of successive reflections made by a particle inside well II prior to exit is $(i-1)$. We will show that the distributions of successive reflection numbers and times obey power laws with same exponent. If $i=1$, the particle has not yet suffered a reflection, but if $i=2$ the particle, still trapped inside well II, has suffered 1 reflection. The time associated with the reflections is $t_{i}^{s}=(i-1) 2 t_{n}^{\prime \prime \prime}$. If condition $(a)$ is satisfied, then the particle is transmitted across the barrier with kinetic energy $K_{n}^{\prime \prime \prime}=E_{n}^{\prime \prime}$ 
$-\left[V_{0}+V_{1} \sin \left(t_{n}+t_{n}^{\prime}+t_{n}^{\prime \prime}+2 i t_{n}^{\prime \prime \prime}\right)\right]$ in a time $t_{n}^{\prime v}=b / v_{n}^{\prime \prime \prime}$ where $v_{n}^{\prime \prime \prime}=\sqrt{2 K_{n}^{\prime \prime \prime} / m}$, exiting the barrier with total energy $E_{n+1}$ $=K_{n}^{\prime \prime \prime}+V_{0}+V_{1} \sin \left(t_{n}+t_{n}^{\prime}+t_{n}^{\prime \prime}+2 i t_{n}^{\prime \prime \prime}+t_{n}^{\prime v}\right)$. Since the total energy of the particle is constant until its next passage across the barrier, $T_{B}$ giving the new energy and the new time for the $(n+1)$ th kick from the wall of infinite potential located at $x$ $=0$ is written as

$$
T_{B}:\left\{\begin{aligned}
E_{n+1}= & E_{n}+V_{1}\left[-\sin \left(t_{n}+t_{n}^{\prime}\right)+\sin \left(t_{n}+t_{n}^{\prime}+t_{n}^{\prime \prime}\right)\right. \\
& -\sin \left(t_{n}+t_{n}^{\prime}+t_{n}^{\prime \prime}+2 i t_{n}^{\prime \prime \prime}\right) \\
& \left.+\sin \left(t_{n}+t_{n}^{\prime}+t_{n}^{\prime \prime}+2 i t_{n}^{\prime \prime \prime}+t_{n}^{\prime v}\right)\right], \\
t_{n+1}= & t_{n}+t_{n}^{\prime}+t_{n}^{\prime \prime}+2 i t_{n}^{\prime \prime \prime}+t_{n}^{\prime v}+l / \sqrt{2 E_{n+1} / m} .
\end{aligned}\right.
$$

We may use the following dimensionless variables: $r$ $=V_{1} / V_{0} ; e_{n}=E_{n} / V_{0} ; e_{n}^{\prime}=K_{n}^{\prime} / V_{0} ; e_{n}^{\prime \prime}=K_{n}^{\prime \prime} / V_{0} ; e_{n}^{\prime \prime \prime}=K_{n}^{\prime \prime \prime} / V_{0} ; \phi_{n}$ $=(2 \pi / \tau) t_{n} ; w=\sqrt{2} V_{0} / m$; and $M=l / w \tau$. Here, $\tau$ gives the period of oscillation of the potential barrier, and the product $w \tau$ gives the distance that a particle travels with velocity $w$ in a time $\tau$. So the parameter $M$ gives the number of oscillations that the oscillating barrier has completed after the particle starts from $x=0$ with kinetic energy $K=V_{0}$ by the time it arrives at the left-hand side of the potential barrier located at $x=l[8,9]$. Using these variables, the application $T_{A}$ is given by

$$
T_{A}:\left\{\begin{array}{l}
e_{n+1}=e_{n}, \\
\phi_{n+1}=\phi_{n}+4 \pi M / \sqrt{e_{n+1}} \bmod (2 \pi),
\end{array}\right.
$$

and the application $T_{B}$ is

$$
T_{B}:\left\{\begin{array}{c}
e_{n+1}=e_{n}+r\left[-\sin \left(\Delta \phi_{a}\right)+\sin \left(\Delta \phi_{b}\right)\right. \\
\left.-\sin \left(\Delta \phi_{c}\right)+\sin \left(\Delta \phi_{d}\right)\right], \\
\phi_{n+1}=\Delta \phi_{d}+2 \pi M / \sqrt{e_{n+1}} \bmod (2 \pi),
\end{array}\right.
$$

where the auxiliaries variables are

$$
\begin{gathered}
\Delta \phi_{a}=\phi_{n}+\frac{2 \pi M}{\sqrt{e_{n}}}, \\
\Delta \phi_{b}=\phi_{n}+2 \pi M\left[\frac{1}{\sqrt{e_{n}}}+\frac{b}{l} \frac{1}{\sqrt{e_{n}^{\prime}}}\right], \\
\Delta \phi_{c}=\phi_{n}+2 \pi M\left[\frac{1}{\sqrt{e_{n}}}+\frac{b}{l} \frac{1}{\sqrt{e_{n}^{\prime}}}+2 i \frac{L}{l} \frac{1}{\sqrt{e_{n}^{\prime \prime}}}\right], \\
\Delta \phi_{d}=\phi_{n}+2 \pi M\left[\frac{1}{\sqrt{e_{n}}}+\frac{b}{l} \frac{1}{\sqrt{e_{n}^{\prime}}}+2 i \frac{L}{l} \frac{1}{\sqrt{e_{n}^{\prime \prime}}}+\frac{b}{l} \frac{1}{\sqrt{e_{n}^{\prime \prime \prime}}}\right] .
\end{gathered}
$$

The expressions for the new energies $e_{n}^{\prime}, e_{n}^{\prime \prime}$, and $e_{n}^{\prime \prime \prime}$ are given by

$$
\begin{aligned}
& e_{n}^{\prime}=e_{n}-1-r \sin \left(\Delta \phi_{a}\right), \\
& e_{n}^{\prime \prime}=e_{n}^{\prime}+1+r \sin \left(\Delta \phi_{b}\right), \\
& e_{n}^{\prime \prime \prime}=e_{n}^{\prime \prime}-1-r \sin \left(\Delta \phi_{c}\right) .
\end{aligned}
$$

The coefficients of the Jacobian matrices for $T_{A}$ and $T_{B}$ applications are shown in the Appendix. They ensure that the

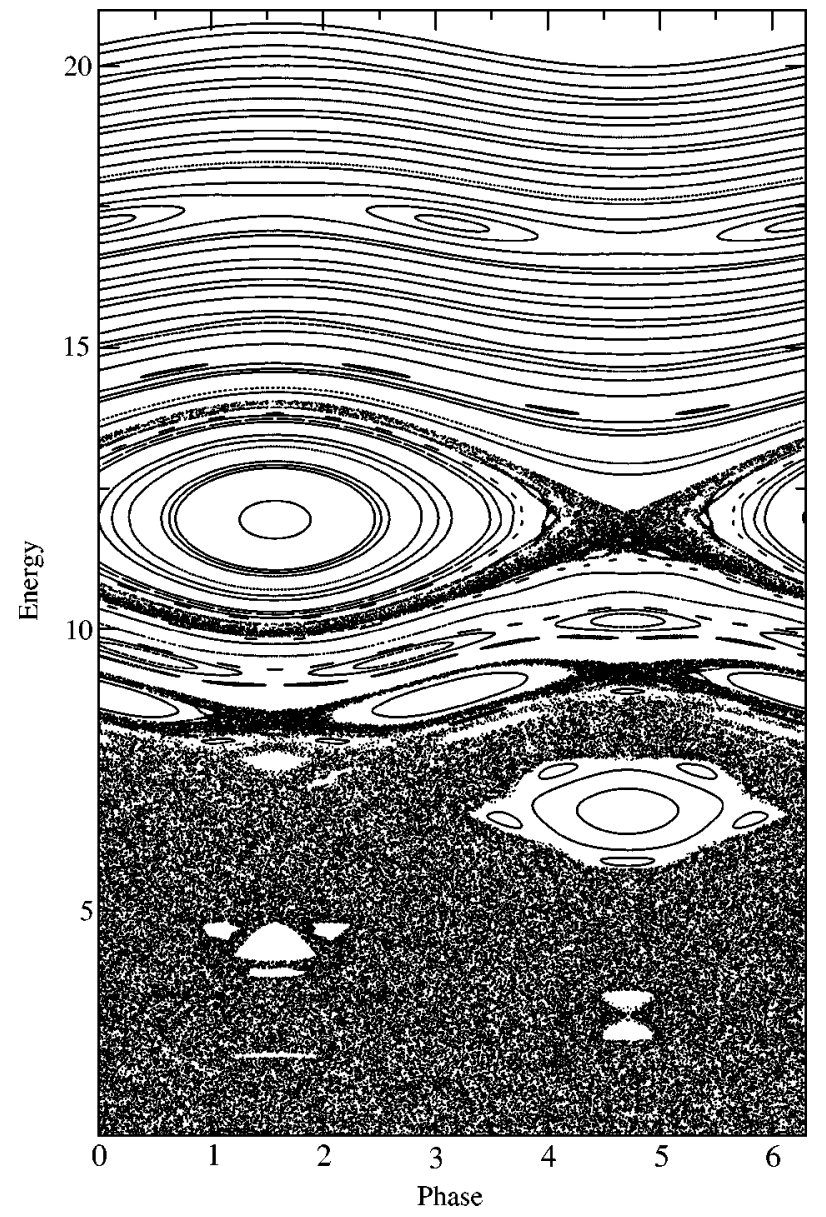

FIG. 2. Iteration of different initial conditions for the parameters $M=4.7, r=0.5, b / l=0.2$, and $L / l=1$. In the low energy regime it is easy to see KAM islands surrounded by a chaotic sea that is limited by one spanning curve. For intermediate energies it is possible to observe a small chaotic region that is enclosed by two different spanning curves. The high energy domain shows many different spanning curves.

maps are area-preserving, because $\operatorname{det} J_{A, B}=1$.

Iteration of $T$ for the parameters $M=4.7, r=0.5, b / l=0.2$ and $L / l=1$ yields the phase space shown in Fig. 2. To facilitate a comparison with previous results in the literature, Fig. 2 was obtained with the same control parameters used in Ref. [9]. In the low energy regime we can see KAM islands surrounded by a chaotic sea. The chaotic sea is limited by the first invariant spanning curve. For intermediate values of energy, we see a small chaotic region that is limited by two different invariant spanning curves. As the map is areapreserving, a chaotic orbit enclosed by two different spanning curves will never exit them and, similarly, a chaotic orbit located below the first invariant spanning curve will never rise above the curve nor visit the interior of a KAM island. For high values of energy, we mostly see invariant spanning curves. We focus our attention on the low energy regime.

The successive reflection number consists basically of the number of the iteration for which the particle is still trapped in well II. It is obtained using the following condition: 

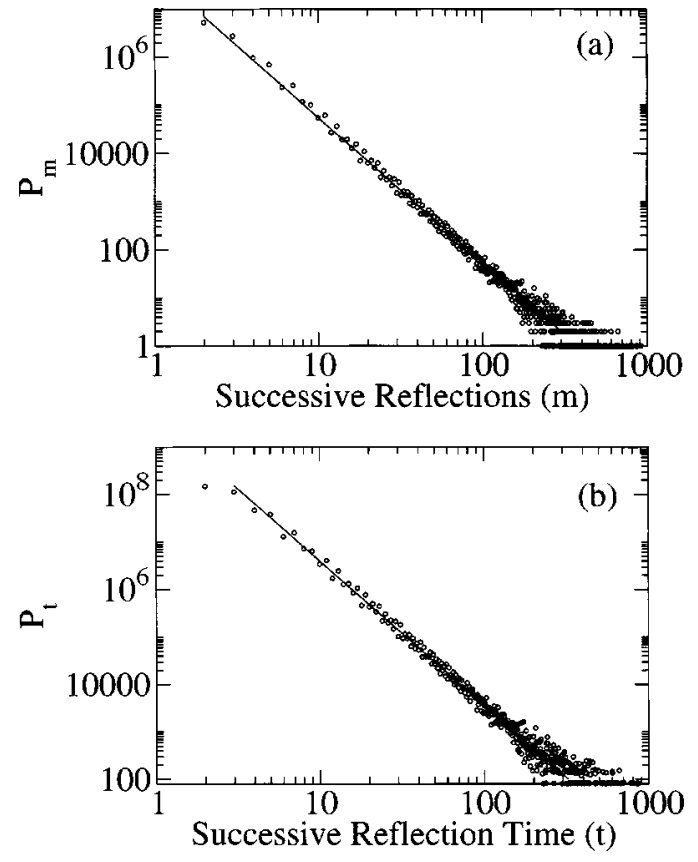

FIG. 3. Distributions of successive reflection (a) numbers and (b) times. The parameters used here are $M=4.7, b / l=L / l=1$ and $r=0.5$. The power law fits yield $P_{m} \propto m^{\gamma_{m}}$ and $P_{t} \propto t^{\gamma_{t}}$, where $\gamma_{m}=$ $-3.03(2)$ and $\gamma_{t}=-3.00(2)$.

$$
e_{n}^{\prime \prime}>1+r \sin \left(\Delta \phi_{b}+i \frac{4 \pi M}{\sqrt{e_{n}^{\prime \prime}}} \frac{L}{l}\right),
$$

where $i$ is the smallest integer for which Eq. (1) is true. Note that for $i>1$, the successive reflection number is given by $m=(i-1)$. As the particle travels at constant velocity inside well II, it is easy to obtain its reflection time as

$$
t_{m}^{s}=m \frac{4 \pi M}{\sqrt{e_{n}^{\prime \prime}}} \frac{L}{l}
$$

The distributions of successive reflection numbers, $P_{m}$, and successive reflection times, $P_{t}$ are shown in Fig. 3. In each case, the straight line represents a power law fit to the numerical data. In Fig. 3(a), the power law fit for $P_{m} \propto m^{\gamma_{m}}$ yields $\gamma_{m}=-3.03(2)$. It is interesting to note that large values of $m$ have low occurrence, and small values of $m$ have a high probability of being observed. The distribution of the average successive times is shown in Fig. 3(b). The power law fit suggests to us that $P_{t} \propto t^{\gamma_{t}}$ with $\gamma_{t}=-3.00(2)$. The control parameters used in Fig. 3 were $M=4.7, r=0.5, b / l=L / l=1$. Note that both distributions are fitted well by power laws with the same exponent of -3 .

\section{B. Lyapunov exponents}

One tool used to characterize sensitivity to initial conditions is the well known Lyapunov exponent. In effect, it corresponds to evolving the system over a long time from two slightly different initial conditions. If the two trajectories diverge exponentially in time, the behavior of the system is considered to be chaotic, the orbit is called chaotic, and the
Lyapunov exponent is positive. If the Lyapunov exponent is negative, the orbit may be either periodic or quasi-periodic. Let us now describe briefly the procedure used to obtain Lyapunov exponents numerically. They are defined $[19,20]$ as

$$
\lambda_{j}=\lim _{n \rightarrow \infty} \sum_{k=1}^{n} \frac{1}{n} \ln \left|\Lambda_{j}^{k}\right|, \quad j=1,2,
$$

where $\Lambda_{j}^{k}$ are the eigenvalues of $M=\prod_{k=1}^{n} J_{k}\left(e_{k}, \phi_{k}\right)$ and $J_{k}$ is the Jacobian matrix evaluated on the orbit $\left(e_{k}, \phi_{k}\right)$. In order to evaluate the eigenvalues of $M$, we use the fact that $J$ can be written as a product of $J=\Theta T$, where $\Theta$ is an orthogonal matrix and $T$ is a triangular one. We now define the elements of these matrices as

$$
\Theta=\left(\begin{array}{cc}
\cos (\theta) & -\sin (\theta) \\
\sin (\theta) & \cos (\theta)
\end{array}\right), \quad T=\left(\begin{array}{cc}
T_{11} & T_{12} \\
0 & T_{22}
\end{array}\right) .
$$

Since $M$ is defined as $M=J_{n} J_{n-1} \ldots J_{2} J_{1}$, we can introduce the identity operator, rewrite $M$ as $M=J_{n} J_{n-1} \ldots J_{2} \Theta_{1} \Theta_{1}^{-1} J_{1}$, and define $\Theta_{1}^{-1} J_{1}=T_{1}$. The product $J_{2} \Theta_{1}$ defines a new matrix $J_{2}^{*}$. In a following step, we may write $M$ as $M$ $=J_{n} J_{n-1} \ldots J_{3} \Theta_{2} \Theta_{2}^{-1} J_{2}^{*} T_{1}$. The same procedure yields $T_{2}$ $=\Theta_{2}^{-1} J_{2}^{*}$. The problem is thus reduced to the evaluation of the diagonal elements of $T_{i}: T_{11}^{i}, T_{22}^{i}$. Using the $\Theta$ and $T$ matrices, we find the eigenvalues of $M$, given by

$$
T_{11}=\frac{j_{11}^{2}+j_{21}^{2}}{\sqrt{j_{11}^{2}+j_{21}^{2}}}, \quad T_{22}=\frac{j_{11} j_{22}-j_{12} j_{21}}{\sqrt{j_{11}^{2}+j_{21}^{2}}} .
$$

We can then evaluate the Lyapunov exponent using the relation

$$
\lambda_{j}=\lim _{n \rightarrow \infty} \sum_{k=1}^{n} \frac{1}{n} \ln \left|T_{j}^{k}\right|, \quad j=1,2 .
$$

It is interesting to observe that $\lambda_{1}=-\lambda_{2}$, because the map is area-preserving. Figure 4 shows the asymptotic convergence of the positive Lyapunov exponent for the same parameters as in Fig. 2. The ensemble average of 5 different initial conditions gives $\bar{\lambda}=1.675 \pm 0.003$, where the error represents the standard deviation of the five samples.

\section{NUMERICAL RESULTS}

\section{A. Lyapunov exponent evaluation}

In this section we present and discuss our numerical results for the deterministic case. Our first step is to choose control parameters, and then we investigate the corresponding dynamical properties. The Lyapunov exponent is evaluated for a large range of control parameters. Figure 5 shows the behavior of the Lyapunov exponent for a large range of $M$. We note that, for three different values of $r(r=0.25, r$ $=0.5$ and $r=0.75$ ) and for two different combinations of $b / l$ and $L / l$ (the symmetrical case $b / l=L / l=1$, and an asymmetrical one $b / l=0.5$ and $L / l=1$ ) the Lyapunov exponent grows slowly and monotonically for 4 decades in the $M$ parameter. Each point in Fig. 5 was obtained by the averaging 


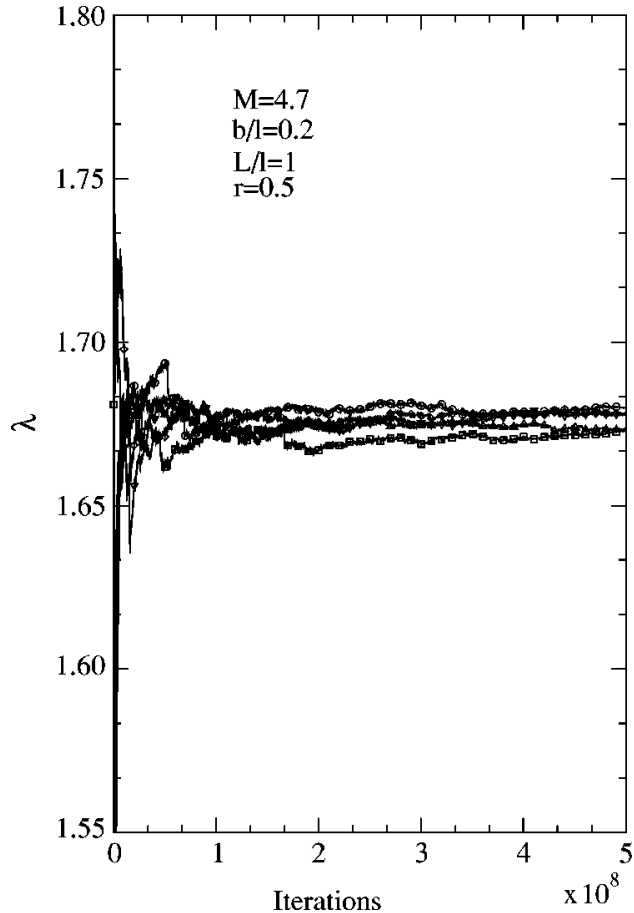

FIG. 4. Convergence of the Lyapunov exponent in the low energy domain. The parameters used are $M=4.7, r=0.5, b / l=0.2$, and $L / l=1$ and the Lyapunov exponent value is $\bar{\lambda}=1.675 \pm 0.003$.

over 5 different initial conditions on the chaotic sea, iterated $5 \times 10^{8}$ times. This iteration number guarantees good convergence of the Lyapunov exponent, as shown in Fig. 4. The error bars indicates the standard deviation of the 5 samples. It is interesting to observe that there are no abrupt transitions in $\lambda$ as a function of $M$ within the huge parameter range studied.

Next we investigate how the Lyapunov exponent varies as a function of $r$. We choose the symmetrical case with fixed $M=4.7$. The behavior of $\lambda$ as a function of $r$ is found to be as shown in Fig. 6. We observe one very pronounced and abrupt transition, and 4 other smaller transitions. These transitions are associated with the destruction of the first invariant spanning curve and the consequent merging of different larger chaotic regions. Each region has its own characteristic Lyapunov exponent. In a recent paper [21] the authors observed a similar transition in the context of a particle confined within an infinite potential box containing an oscillating square well [22]. They proposed that, after the transition, the Lyapunov exponent is given by an average of the previous exponents scaled by the relative sizes of the corresponding regions of the phase space. We emphasize that this conjecture works well here too. To illustrate this, let us use the most pronounced peak, which occurs near $r=0.0075$. Before the transition, it is easy to observe different chaotic regions that are separated by an invariant spanning curve, as is shown in Fig. 7(a). The Lyapunov exponents for regions below and above the first invariant spanning curve are $\lambda_{b}$ $=4.44 \pm 0.06$ and $\lambda_{a}=0.811 \pm 0.001$, respectively. These Lyapunov exponent values were obtained from a long run of $2 \times 10^{9}$ iterations using 10 different initial conditions. A naive estimation of fraction occupied is $f_{b}=0.33$ below, and
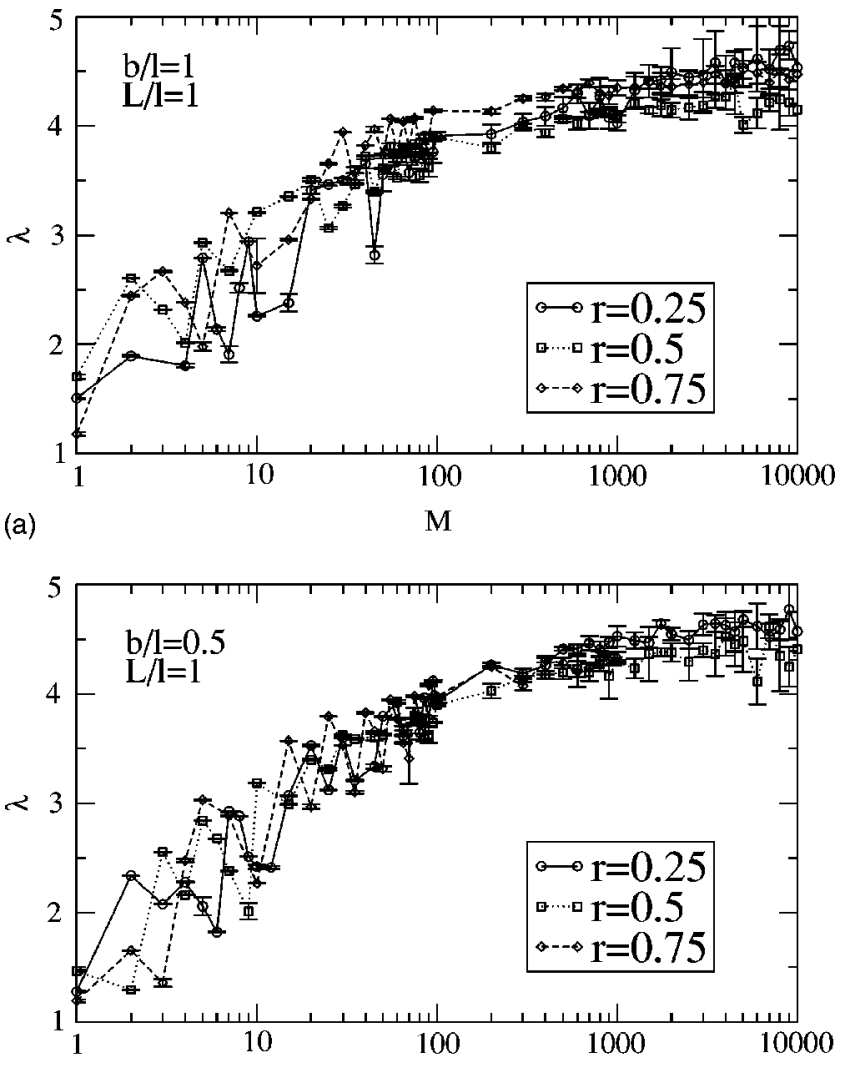

(b)

$\mathrm{M}$

FIG. 5. Log linear plot of the Lyapunov exponent $\lambda$ as a function of $M$. The parameters used are $r=0.25, r=0.5, r=0.75, L / l$ $=1$ and (a) $b / l=1$, i.e., the symmetrical case; and (b) $b / l=0.5$, i.e., the asymmetrical case.

$f_{a}=0.67$ above. After the transition, just one initial condition is sufficient to fill the chaotic region, now representing a merging of regions $I$ and $I I$, as can be seen in Fig. 7(b). Using the relation proposed in [21], we can estimate the Lyapunov exponent after the transition from the values of the Lyapunov exponent in the two different chaotic regions prior to the transition as

$$
\bar{\lambda}=f_{a} \lambda_{a}+f_{b} \lambda_{b}=2.008,
$$

that is in a good accord with Fig. 6. It is interesting to note that, after the transition, the Lyapunov exponent convergence is slower and the error bars are larger. The other transitions shown in Fig. 6 may be characterized in the same way.

\section{B. Resonances on the chaotic sea}

Let us now discuss the distribution of successive reflection energies. The range of energies that allow the particle to stay trapped is $e \in\left[e_{\min }, e_{\max }\right]$, where $e_{\min }=1-r$ and $e_{\max }$ $=1+r$. This is the same range of energies within which the phenomenon of resonance occurs. Resonance is associated with the time that the particle spends traveling inside well II, 


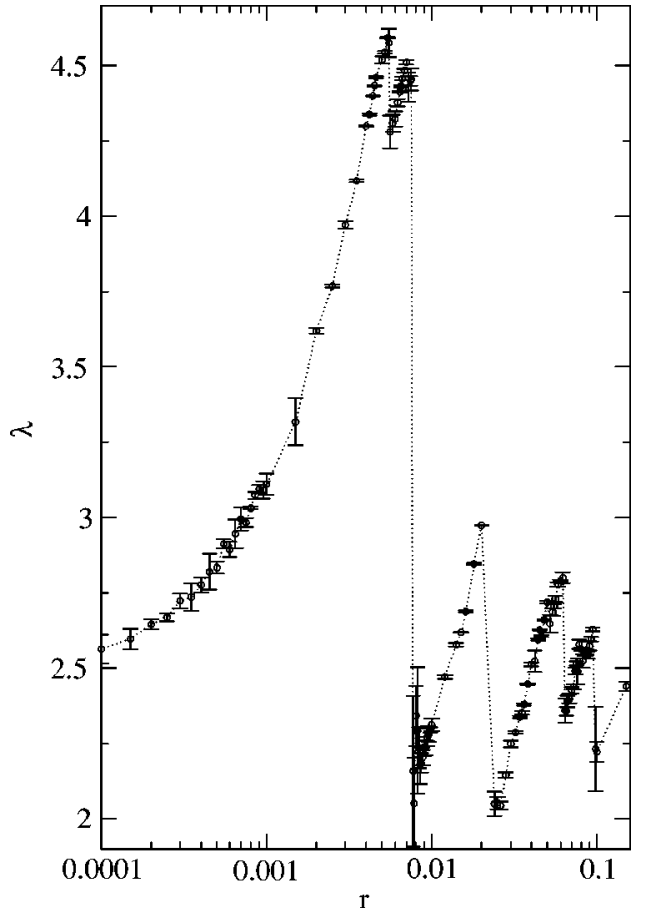

FIG. 6. Log linear plot of the variation of the Lyapunov exponent $\lambda$ with $r$. The parameters used were $M=4.7$ and $b / l=L / l=1$.

$$
t^{\prime \prime \prime}=\frac{i 4 \pi M}{\sqrt{e^{\prime \prime}}} \frac{L}{l} .
$$

If this time is a multiple of $2 \pi$, the particle will not be trapped within well II and $i$ takes the value $i=1$. It is possible to estimate the number of oscillations of the barrier for this range of energy since we have that

$$
\frac{4 \pi M}{\sqrt{e^{\prime \prime}}} \frac{L}{l}=2 k \pi
$$

where $k$ gives the number of oscillations of the potential barrier. The minimum and maximum values of $k$ for the range $e \in\left[e_{\text {min }}, e_{\text {max }}\right]$ is

$$
k_{\text {min }}=\frac{2 M}{\sqrt{e_{\text {max }}^{\prime \prime}}} \frac{L}{l}, \quad k_{\text {max }}=\frac{2 M}{\sqrt{e_{\text {min }}^{\prime \prime}}} \frac{L}{l} .
$$

For the symmetrical case with $M=4.7, r=0.5$, we find that $k_{\text {min }}=8$ and $k_{\text {max }}=13$. The corresponding energies and times of flight, $t_{f}$, within well II are shown in Table I. The distribution of successive energies in well II is shown in Fig. 8. The resonance energies are indicated in the figure.

\section{Scaling properties}

Let us now discuss the main idea of scaling properties for chaotic time series in this problem. Figure 9 shows the evolution of one chaotic time series for the parameters $b / l$ $=L / l=1, r=0.5$, and $M=4.7$. The amplifications in Fig. 9 allow us to suppose that some scaling property can be derived from this time series, once that it has basically the same behavior for very different time scales. We choose to
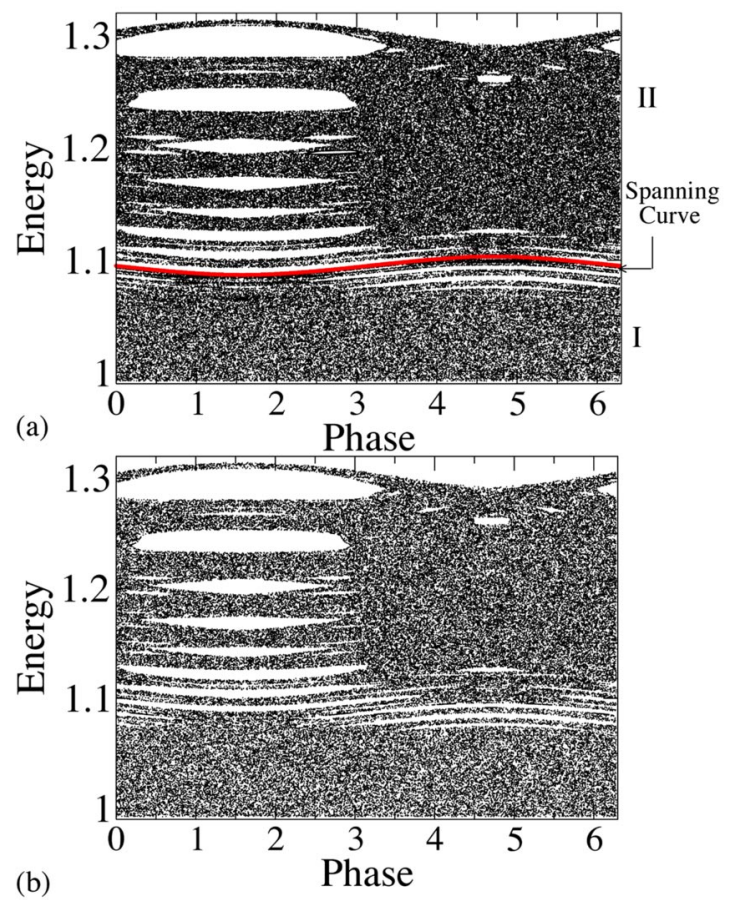

FIG. 7. (a) Iteration of three different initial conditions that lie, respectively, (i) below, (ii) inside, and (iii) above the first invariant spanning curve. (b) Iteration of only one initial condition. The parameters used were $M=4.7, b / l=L / l=1$ and (a) $r=0.0075$ and (b) $r=0.0076$.

characterize the behavior in terms of the variance of the average energy, which we will refer to as the roughness, $\omega$ [23]. The scaling does not work well for the dependence on $r$ because, as $r$ varies, it is easy to identify where merging takes place between the different large chaotic regions (see Figs. 6 and 7). Our results clearly work well for the dependence on $M$, however, since merging events between the large chaotic regions are not observed. We choose to study the same range of $M$ as in Fig. 5, just excluding the first decade. We consider the case of a symmetrical system and set $r=0.1$, but similar behavior can, in fact, be observed for other values of $r$.

Given the time series, it is easy to evaluate both average and average squared energies, defined, respectively, as

TABLE I. Resonance energies and flight times inside well II for the control parameters $M=4.7, r=0.5$ and $b / l=L / l=1$.

\begin{tabular}{rlc}
\hline \hline$k$ & \multicolumn{1}{c}{$e_{k}^{\prime \prime}$} & $t_{f}$ \\
\hline 8 & $1.3806 \ldots$ & $50.2654 \ldots$ \\
9 & $1.0908 \ldots$ & $56.5486 \ldots$ \\
10 & 0.8836 & $62.8318 \ldots$ \\
11 & $0.7302 \ldots$ & $69.1150 \ldots$ \\
12 & $0.6136 \ldots$ & $75.3982 \ldots$ \\
13 & $0.5228 \ldots$ & $81.6814 \ldots$ \\
\hline \hline
\end{tabular}




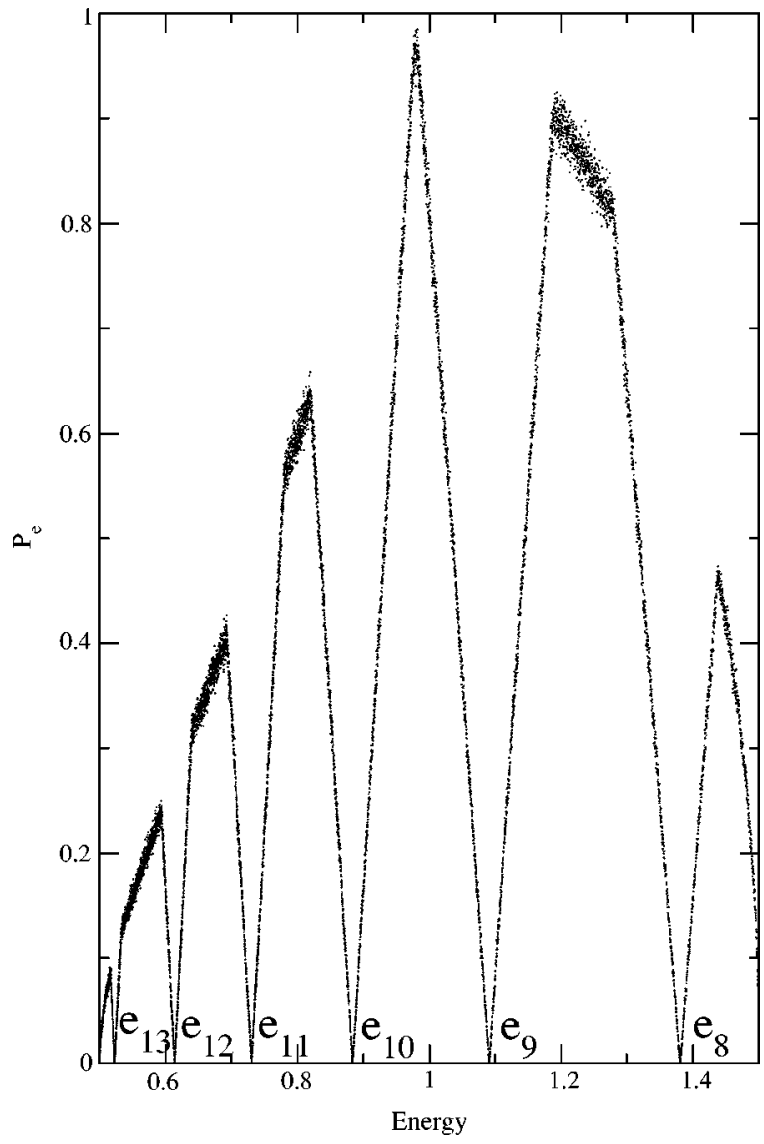

FIG. 8. Normalized distribution of successive reflection energies for parameters $M=4.7, r=0.5, b / l=L / l=1$.

$$
\bar{e}(N, M)=\frac{1}{N} \sum_{n=1}^{N} e_{n}, \quad \bar{e}^{2}(N, M)=\frac{1}{N} \sum_{n=1}^{N} e_{n}^{2} .
$$

After that, we can define the roughness as

$$
\omega(N, M) \equiv \frac{1}{B} \sum_{j=1}^{B} \sqrt{\bar{e}_{j}^{2}(N, M)-\bar{e}_{j}^{2}(N, M)},
$$

where we use an ensemble of $B$ different initial conditions. The average over $B$ is used in order to smooth the roughness evolution. Figure 10 shows one roughness evolution. It was constructed using an ensemble of 10000 different initial phases and the same initial energy $e=1+r$, all of them giving rise to chaotic evolution. We can see in Fig. 10 two different kinds of behavior as a function of the iteration number. Following a brief initial transient, the roughness grows according to a power law, but it eventually reaches a regime of saturation for a large enough number of iterations. The change in behavior is characterized by a typical crossover iteration number $N_{x}$, as shown in Fig. 10.

This saturation of the roughness curves is a consequence of the limited time series amplitude. It is well known that the amplitude depends on the position of the first invariant spanning curve since, in the deterministic model, the chaotic sea is limited by such a spanning curve. For the range of control parameter studied (see Fig. 11), one effect of increasing $M$ is
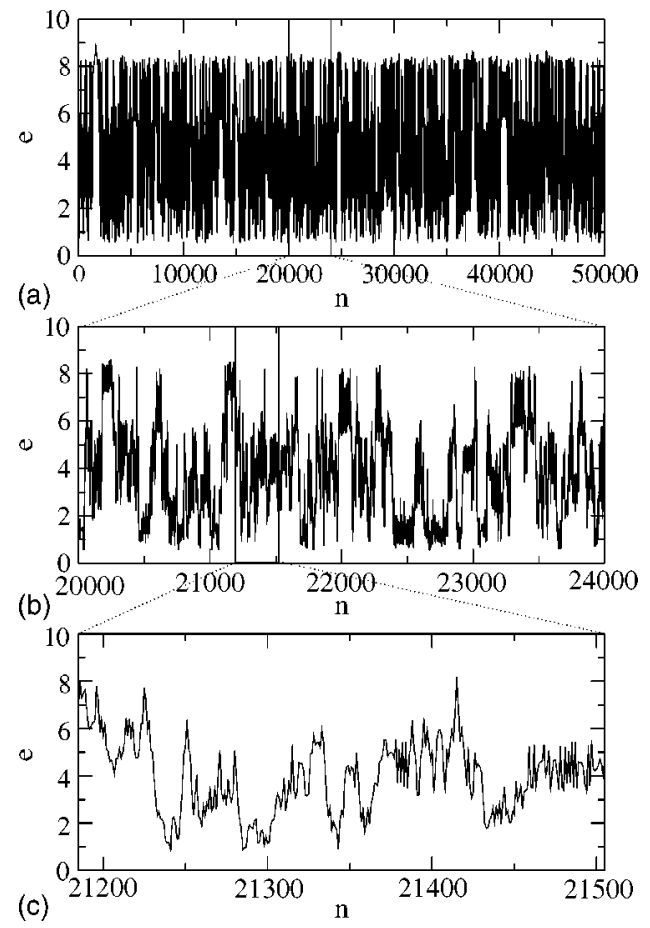

FIG. 9. The time evolution for one initial condition located in the chaotic sea for parameters $M=4.7 ; r=0.5 ; b / l=L / l=1$.

to localize the first invariant spanning curve in a higher position. As an immediate consequence of this, the variance will saturate at a higher value. Thus, we can start to study the roughness scaling, supposing following:

(i) After the brief initial transient, the roughness grows as function of iteration number according to

$$
\omega(N, M) \propto N^{\beta} \text {. }
$$

This growth can be seen in region I of Fig. 10. $\beta$ is called the growth exponent. Equation (2) is valid for $N \ll N_{x}$.

(ii) As the iteration number increases, the roughness reaches saturation, as can be seen in region II of Fig. 10. The behavior of the roughness within the saturation regime follows the equation

$$
\omega_{\text {sat }}(N, M) \propto M^{\alpha},
$$

where $\alpha$ is the roughening exponent. Equation (3) is valid just for $N \gg N_{x}$.

(iii) The crossover iteration number $N_{x}$ that tells us when the roughness growth slows and saturation is approached is given by

$$
N_{x}(M) \propto M^{z} .
$$

Here $z$ is called the dynamical exponent.

Let now us discuss the procedure used to obtain the saturation value. Even for a maximum iteration number of $1000 N_{x}$, our simulation exhibits a final roughness evolution showing that it has still not reached saturation. To solve this problem, we can choose two different options. The first one is increase the iteration number yet more, and start all simulations again. This choice obviously carries the disadvantage of very much longer simulations. The second, and more de- 


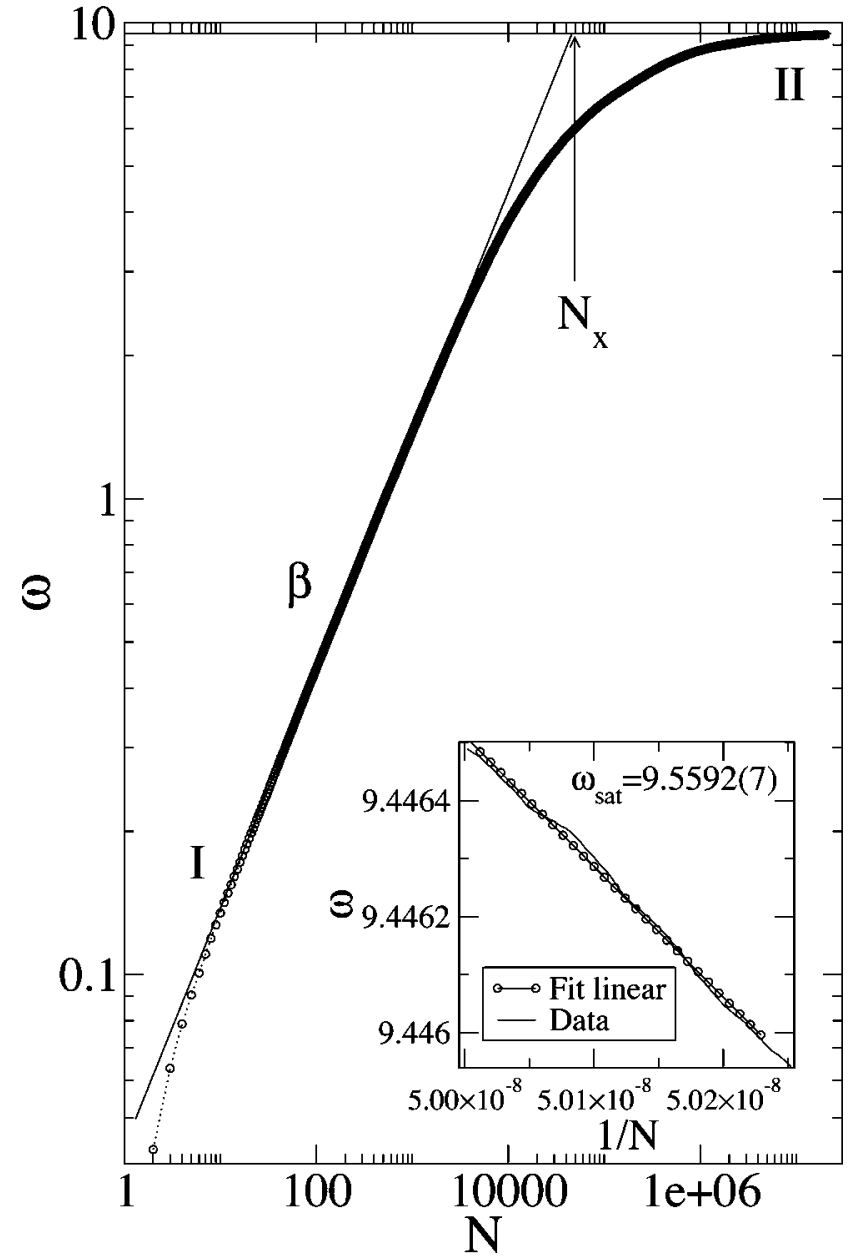

FIG. 10. Roughness behavior for an ensemble of 10000 different initial phases $\phi$ and the same initial energy $e=1+r$. Both initial conditions give rise to chaotic orbits. The inset shows the procedure used to obtain the roughness in the saturation regime after applying the transformation $N \rightarrow 1 / N$.

sirable, option is to extrapolate the saturation roughening to an infinite iteration number. It consists basically in applying the following transformation to the iteration number $N$ $\rightarrow 1 / N$, and it is applicable because the saturation increases slowly and linearly for sufficiently large values of $N$. After performing this transformation, the saturation roughness is given by

$$
\omega(N, M)=\omega_{\text {sat }}(M)+\frac{b^{\prime}}{N} .
$$

When $N \rightarrow \infty$ in Eq. (5), we have that $\omega(\infty, M) \rightarrow \omega_{\text {sat }}$. Technically, the saturation roughness can be obtained as the linear coefficient of Eq. (5), given by linear regression. This procedure is shown in the inset of Fig. 10.

Next, we obtain the intercept of the power law (in region I of Fig. 10) with the saturation value obtained from Eq. (5). In order to obtain the exponents $\alpha$ and $z$, we plot $\omega_{\text {sat }} \times M$ and $N_{x} \times M$, as shown in Fig. 11. These power law fits yield $\alpha=0.670(1)$ and $z=1.331(6)$. The mean $\beta=0.500(6)$ is obtained from an average of all the roughness curves.
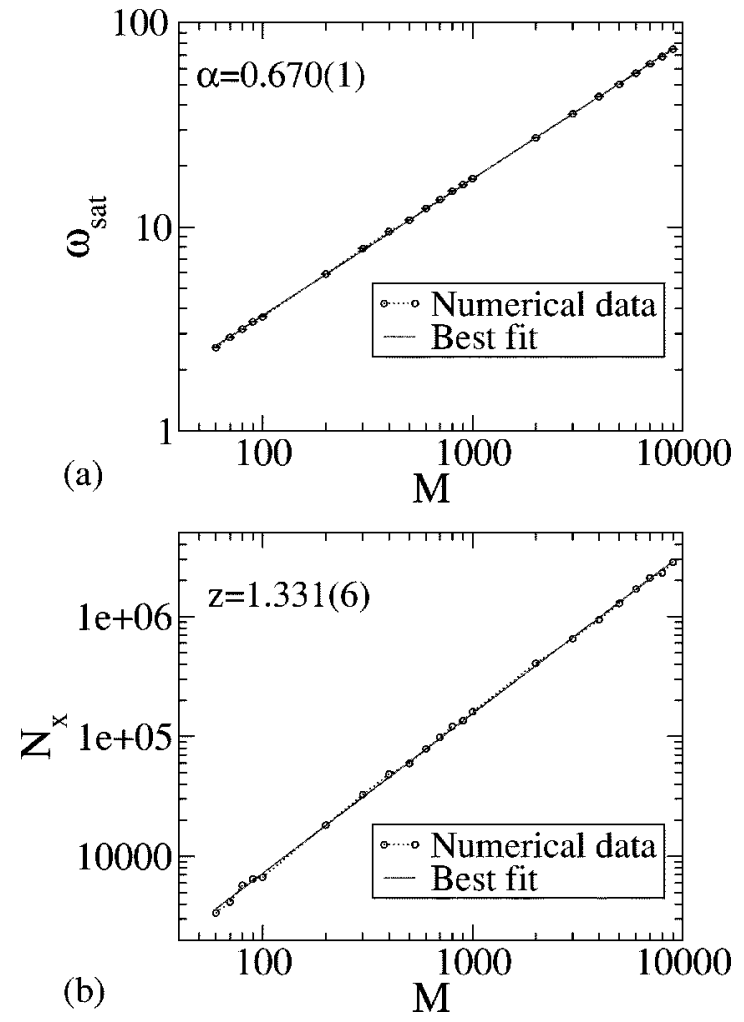

FIG. 11. The dependencies on $M$ of (a) the saturation roughness $\omega_{\text {sat }}$ and (b) the crossover iteration number $N_{x}$.

We can now proceed to "collapse" the roughness curves onto a universal plot. The first step is to take the ratio $\omega(N, M) / \omega_{\text {sat }}(M)$. This relocates all curves to the same saturation value, as can be seen in Fig. 12(b). The second step is to take the ratio $N / N_{x}$. It collapses the curves onto the same characteristic iteration number, as can be seen in Fig. 12(c).

The success of these procedures in producing a single, universal, roughness plot allows us to describe the roughness according to the following scaling function:

$$
\omega(N, M)=\zeta \omega\left(\zeta^{a} N, \zeta^{c} M\right),
$$

where $\zeta$ is the scaling factor. Choosing $\zeta=N^{-1 / a}$, we obtain

$$
\omega(N, M)=N^{-1 / a} \omega_{1}\left(N^{-c / a} M\right) .
$$

The function $\omega_{1}\left(N^{-c / a} M\right)=\omega\left(1, N^{-c / a} M\right)$ and it is supposed to be constant for $N \ll N_{x}$. Using Eq. (2), we can obtain

$$
N^{-1 / a}=N^{\beta},
$$

where $\beta=-1 / a$. From our numerical simulations, we find that $\beta=0.500(6)$.

The second step is to choose $\zeta=M^{-1 / c}$ and it follows that

$$
\omega(N, M)=M^{-1 / c} \omega_{2}\left(M^{-a / c} N\right) .
$$

Here, the function $\omega_{2}\left(M^{-a / c} N\right)=\omega\left(M^{-a / c} N, 1\right)$ and it is supposed to be constant for $N \gg N_{x}$. Using the equation (3), we obtain that

$$
M^{-1 / c}=M^{\alpha},
$$

and we have $\alpha=-1 / c$. 
From a combination of equations $\zeta=N^{-1 / a}, \zeta=M^{-1 / c}$ and (4) and using conditions (6) and (7) it is easy to show that $M^{z \beta} \propto M^{\alpha}$. Thus, we find that there is a relationship between the critical exponents given by

$$
z=\frac{\alpha}{\beta}
$$

Using the numerical results for $\alpha$ and $\beta$ and evaluating Eq. (8), we are able to compare the result with the value of $z$ obtained from the numerical fit shown in Fig. 11(b). From (8) we obtain $z=1.34(1)$, which is gratifyingly close to the numerical value of $z=1.331(6)$ obtained from Fig. 11(b).

\section{STOCHASTIC VERSION}

Next, we discuss the stochastic version of the problem. We suppose that the potential $V_{1}(x, t)$ is written as $V_{1}(x) f(t)$, where $f(t)$ gives us random numbers uniformly distributed in $[-1,1]$. The variables are now total energy and time, and the applications are given by

$$
T_{A}:\left\{\begin{array}{l}
e_{n+1}=e_{n}, \\
t_{n+1}=t_{n}+4 \pi M / \sqrt{e_{n+1}},
\end{array}\right.
$$

if $e_{n}<1+r f\left(t_{n}+2 \pi M / \sqrt{e_{n}}\right)$, otherwise, the application $T_{B}$ is

$$
T_{B}:\left\{\begin{array}{l}
e_{n+1}=e_{n}+r\left[f\left(\Delta \phi_{a}\right)+f\left(\Delta \phi_{b}\right)+f\left(\Delta \phi_{c}\right)+f\left(\Delta \phi_{d}\right)\right], \\
t_{n+1}=\Delta \phi_{d}+2 \pi M / \sqrt{e_{n+1}},
\end{array}\right.
$$

where

$$
\begin{gathered}
\Delta \phi_{a}=t_{n}+\frac{2 \pi M}{\sqrt{e_{n}}}, \\
\Delta \phi_{b}=t_{n}+2 \pi M\left[\frac{1}{\sqrt{e_{n}}}+\frac{b}{l} \frac{1}{\sqrt{e_{n}^{\prime}}}\right], \\
\Delta \phi_{c}=t_{n}+2 \pi M\left[\frac{1}{\sqrt{e_{n}}}+\frac{b}{l} \frac{1}{\sqrt{e_{n}^{\prime}}}+2 i \frac{L}{l} \frac{1}{\sqrt{e_{n}^{\prime \prime}}}\right], \\
\Delta \phi_{d}=t_{n}+2 \pi M\left[\frac{1}{\sqrt{e_{n}}}+\frac{b}{l} \frac{1}{\sqrt{e_{n}^{\prime}}}+2 i \frac{L}{l} \frac{1}{\sqrt{e_{n}^{\prime \prime}}}+\frac{b}{l} \frac{1}{\sqrt{e_{n}^{\prime \prime \prime}}}\right],
\end{gathered}
$$

where the energies $e_{n}^{\prime}, e_{n}^{\prime \prime}, e_{n}^{\prime \prime \prime}$ are

$$
\begin{aligned}
& e_{n}^{\prime}=e_{n}-1+r f\left(\Delta \phi_{a}\right), \\
& e_{n}^{\prime \prime}=e_{n}^{\prime}+1+r f\left(\Delta \phi_{b}\right), \\
& e_{n}^{\prime \prime \prime}=e_{n}^{\prime \prime}-1+r f\left(\Delta \phi_{c}\right) .
\end{aligned}
$$

It is interesting to note that $i$ is the smallest integer number for which the equation

$$
e_{n}^{\prime \prime}>1+r f\left(\Delta \phi_{b}+i \frac{4 \pi M}{\sqrt{e_{n}^{\prime \prime}}} \frac{L}{l}\right)
$$

is true.
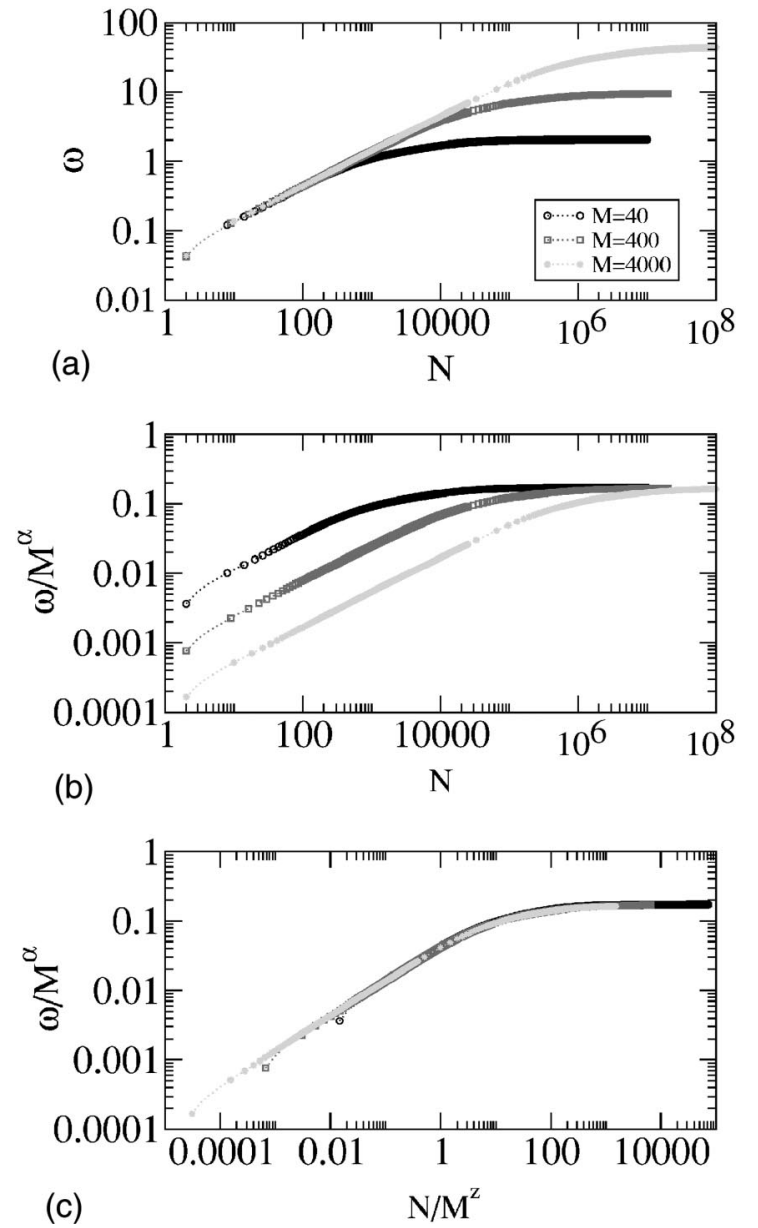

FIG. 12. (a) Roughness evolutions for different $M$. (b) Collapse of the curves onto the same saturation value. (c) Their collapse onto both the same saturation value and the same characteristic crossover iteration number.

Iterating the applications $T_{A}$ and $T_{B}$ for the stochastic version we may study the behavior of the energy as a function of the iteration number and time. It is interesting to observe that $n$ and $t$ are not proportional to each other, since a particle of high energy is described with a larger number of iterations $n$ than a particle of lower energy in the same interval of time. We evaluate the average energy for an ensemble of particles with the same initial energy $e_{0}=1$. We find that the total energy grows as $e \propto n^{\delta_{n}}$ where $\delta_{n}=0.496 \pm 0.001$ and $e \propto t^{\delta_{t}}$ with $\delta_{t}=0.649 \pm 0.001$. Figure 13 shows the energy growth for an ensemble of 10000 particles with initial energy $e_{0}$ $=1$.

\section{SUMMARY AND CONCLUSIONS}

We have considered the classical problem of a particle interacting with a time-modulated potential barrier in two different circumstances: (i) deterministic and (ii) stochastic. In the deterministic case, where we used an area-preserving map $T$ to study some dynamical properties of this model, we found that the phase space has a complex structure including KAM islands surrounded by a chaotic sea that is limited by 

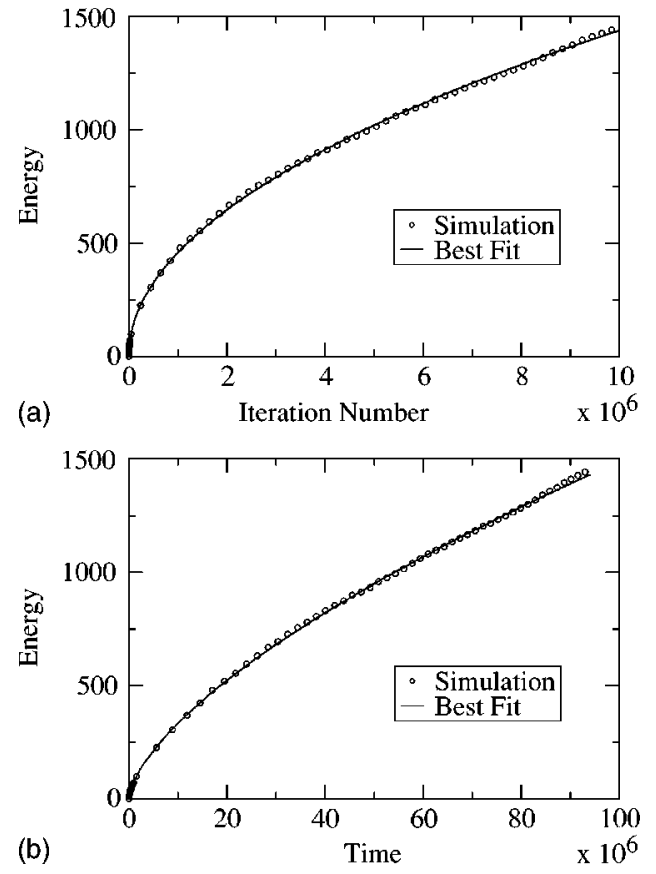

FIG. 13. Average total energy as a function of (a) $n$ and (b) $t$. The parameters used here are $M=4.7, r=0.5, b / l=L / l=1$. Note that $b / l=L / l=1$ is the symmetrical case. The power law gives us that $e \propto n^{\delta_{n}}$ and $e \propto t^{\delta_{t}}$, where $\delta_{n}=0.496 \pm 0.001$ and $\delta_{t}=0.649 \pm 0.001$.

the first invariant spanning curve. Lyapunov exponents were used to characterize the chaotic sea below the first invariant spanning curve for a large range of control parameters. The behavior of $\lambda$ as a function of $M$ for both the symmetrical and asymmetrical cases shows a slow growth in the Lyapunov exponent; similar behavior does not occur for $\lambda$ as a function of $r$. We analyzed the behavior of the roughness as function of $M$ for a chaotic time series located in the chaotic sea. Our results indicates that the roughness obeys a scaling function with very well defined exponents. After a brief transient, the roughness grows with iteration number according to a power law of exponent $\beta=0.500(6)$. After passing a characteristic crossover iteration number, however, the roughness growth slows and approaches a saturation regime. The crossover iteration number increases with the control parameter $M$ according to a power law with a dynamical exponent $z=1.331(6)$. The regime of saturation also depends on the control parameters and it too increases according to a power law as $M$ increases. The roughening exponent is obtained as $\alpha=0.670(1)$. The two exponents are closely related via a scaling function. We found that $\lambda \times r$ exhibits abrupt transitions as the result of a merging of different chaotic regions of varying size. We were able to obtain the Lyapunov exponent after the transition by making use of a conjecture proposed in [21]. It uses the Lyapunov exponent before the transition in the two different chaotic regions scaled by the fraction of the phase space that they occupy. It is especially gratifying that this conjecture still gave good results here too. Depending on the energy of the particle in the chaotic sea, it is possible for it to stay trapped in well II for some interval of time. The distribution of successive reflection numbers, $P_{m}$, and the corresponding distribution of the average succes- sive reflection times, $P_{t}$, are very well fitted by a power law tail. Our results indicates that $P_{m} \propto m^{\gamma_{m}}$ and $P_{t} \propto t^{\gamma_{t}}$ with exponents $\gamma_{m}=\gamma_{t} \sim-3$. These results, like those shown in $[8,9,21]$ may be an indication of some kind of universality class.

For the stochastic version, our results indicate that, after all invariant spanning curves are broken, it is possible to observe unlimited energy growth. The behavior of the average energy for an ensemble of different initial conditions indicates that $e \propto n^{\delta_{n}}$ with $\delta_{n} \sim 0.5$ and $e \propto t^{\delta_{t}}$ with $\delta_{t} \sim 2 / 3$. These exponents are different because a particle of high energy is described by more iterations than a slow one in the same interval of time.

\section{ACKNOWLEDGMENTS}

E. D. Leonel would like to thank Dr. J. K. L. da Silva for valuable discussions. This research was supported by a grant from Conselho Nacional de Desenvolvimento Científico $\mathrm{CNPq}$, from Brazil. The numerical results were obtained partly in CENAPAD-MG/CO in Brazil and partly in the Center for High Performance Computing in Lancaster University. The work was supported in part by the Engineering and Physical Sciences Research Council (UK).

\section{APPENDIX: JACOBIAN COEFFICIENTS}

The coefficients for the Jacobian matrices $J_{A}$ and $J_{B}$ that refer to the applications $T_{A}$ and $T_{B}$, respectively, for the periodic oscillation are given by

$$
\begin{gathered}
j_{11}^{A}=\frac{\partial e_{n+1}}{\partial e_{n}}=1, \quad j_{12}^{A}=\frac{\partial e_{n+1}}{\partial \phi_{n}}=0, \\
j_{21}^{A}=\frac{\partial \phi_{n+1}}{\partial e_{n}}=-\frac{2 \pi M}{e_{n+1}^{3 / 2}}, \quad j_{22}^{A}=\frac{\partial \phi_{n+1}}{\partial \phi_{n}}=1, \\
j_{11}^{B}=\frac{\partial e_{n+1}}{\partial e_{n}}=1-r\left[\cos \left(\Delta \phi_{a}\right) \frac{\partial \Delta \phi_{a}}{\partial e_{n}}-\cos \left(\Delta \phi_{b}\right) \frac{\partial \Delta \phi_{b}}{\partial e_{n}}\right. \\
\left.+\cos \left(\Delta \phi_{c}\right) \frac{\partial \Delta \phi_{c}}{\partial e_{n}}-\cos \left(\Delta \phi_{d}\right) \frac{\partial \Delta \phi_{d}}{\partial e_{n}}\right], \\
j_{12}^{B}=\frac{\partial e_{n+1}}{\partial \phi_{n}}=r\left[-\cos \left(\Delta \phi_{a}\right) \frac{\partial \Delta \phi_{a}}{\partial \phi_{n}}+\cos \left(\Delta \phi_{b}\right) \frac{\partial \Delta \phi_{b}}{\partial \phi_{n}}\right. \\
\left.-\cos \left(\Delta \phi_{c}\right) \frac{\partial \Delta \phi_{c}}{\partial \phi_{n}}+\cos \left(\Delta \phi_{d}\right) \frac{\partial \Delta \phi_{d}}{\partial \phi_{n}}\right], \\
j_{21}^{B}=\frac{\partial \phi_{n+1}}{\partial e_{n}}=\frac{\partial \Delta \phi_{d}}{\partial e_{n}}-\frac{\pi M}{e_{n+1}^{3 / 2}} \frac{\partial e_{n+1}}{\partial e_{n}}, \\
\frac{\partial \phi_{n+1}}{\partial}=1+\frac{\partial \Delta \phi_{d}}{\partial \phi_{n}}-\frac{\pi M}{e_{n+1}^{3 / 2}} \frac{\partial e_{n+1}}{\partial \phi_{n}},
\end{gathered}
$$

where the partial derivatives to the phases are

$$
\frac{\partial \Delta \phi_{a}}{\partial e_{n}}=-\frac{\pi M}{e_{n}^{3 / 2}},
$$




$$
\begin{gathered}
\frac{\partial \Delta \phi_{b}}{\partial e_{n}}=\frac{\partial \Delta \phi_{a}}{\partial e_{n}}-\pi M \frac{b}{l} \frac{1}{e_{n}^{\prime 3 / 2}} \frac{\partial e_{n}^{\prime}}{\partial e_{n}}, \\
\frac{\partial e_{n}^{\prime}}{\partial e_{n}}=1-r \cos \left(\Delta \phi_{a}\right) \frac{\partial \Delta \phi_{a}}{\partial e_{n}}, \\
\frac{\partial \Delta \phi_{c}}{\partial e_{n}}=\frac{\partial \Delta \phi_{b}}{\partial e_{n}}-2 \pi M i \frac{L}{l} \frac{1}{e_{n}^{\prime \prime 3}} \frac{\partial e_{n}^{\prime \prime}}{\partial e_{n}}, \\
\frac{\partial e_{n}^{\prime \prime}}{\partial e_{n}}=\frac{\partial e_{n}^{\prime}}{\partial e_{n}}+r \cos \left(\Delta \phi_{b}\right) \frac{\partial \Delta \phi_{b}}{\partial e_{n}}, \\
\frac{\partial \Delta \phi_{d}}{\partial e_{n}}=\frac{\partial \Delta \phi_{c}}{\partial e_{n}}-\pi M \frac{b}{l} \frac{1}{e_{n}^{\prime \prime \prime 3 / 2}} \frac{\partial e_{n}^{\prime \prime \prime}}{\partial e_{n}}, \\
\frac{\partial e^{\prime \prime \prime}}{\partial e_{n}}=\frac{\partial e_{n}^{\prime \prime}}{\partial e_{n}}-r \cos \left(\Delta \phi_{c}\right) \frac{\partial \Delta \phi_{c}}{\partial e_{n}}, \\
\frac{\partial \Delta \phi_{n}}{\partial}=1,
\end{gathered}
$$

$$
\begin{gathered}
\frac{\partial \Delta \phi_{b}}{\partial \phi_{n}}=\frac{\partial \Delta \phi_{a}}{\partial \phi_{n}}-\pi M \frac{b}{l} \frac{1}{e_{n}^{\prime 3 / 2}} \frac{\partial e_{n}^{\prime}}{\partial \phi_{n}}, \\
\frac{\partial e_{n}^{\prime}}{\partial \phi_{n}}=-r \cos \left(\Delta \phi_{a}\right) \frac{\partial \Delta \phi_{a}}{\partial \phi_{n}} \\
\frac{\partial \Delta \phi_{c}}{\partial \phi_{n}}=\frac{\partial \Delta \phi_{b}}{\partial \phi_{n}}-2 \pi M i \frac{L}{l} \frac{1}{e_{n}^{\prime \prime 3}} \frac{\partial e_{n}^{\prime \prime}}{\partial \phi_{n}} \\
\frac{\partial e_{n}^{\prime \prime}}{\partial \phi_{n}}=\frac{\partial e_{n}^{\prime}}{\partial \phi_{n}}+r \cos \left(\Delta \phi_{b}\right) \frac{\partial \Delta \phi_{b}}{\partial \phi_{n}} \\
\frac{\partial \Delta \phi_{d}}{\partial \phi_{n}}=\frac{\partial \Delta \phi_{c}}{\partial \phi_{n}}-\pi M \frac{b}{l} \frac{1}{e_{n}^{\prime \prime \prime 3 / 2}} \frac{\partial e_{n}^{\prime \prime \prime}}{\partial \phi_{n}} \\
\frac{\partial e_{n}^{\prime \prime \prime}}{\partial \phi_{n}}=\frac{\partial e_{n}^{\prime \prime}}{\partial \phi_{n}}-r \cos \left(\Delta \phi_{c}\right) \frac{\partial \Delta \phi_{c}}{\partial \phi_{n}}
\end{gathered}
$$

These entangled coefficients show that the applications $T_{A}$ and $T_{B}$ preserve the phase space area, because det $J_{A, B}=1$.
[1] C. Cohen-Tannoudji, B. Diu, and F. Laloë, Quantum Mechanics, Vol. I (Wiley, New York, 1977); S. Gasiorowicz, Quantum Physics (Wiley, New York, 1974).

[2] M. Buttiker and R. Landauer, Phys. Rev. Lett. 49, 1739 (1982).

[3] M. Wagner, Phys. Rev. B 57, 11899 (1998).

[4] L. P. Kouwenhoven, S. Jauhar, J. Orenstein and P. L. McEuen, Y. Nagamune, J. Motohisa, and H. Sakaki, Phys. Rev. Lett. 73, 3443 (1994).

[5] P. S. S. Guimarães, B. J. Keay, J. P. Kaminski, S. J. Allen, Jr., P. F. Hopkins, A. C. Gossard, L. T. Florez, and J. P. Harbison, Phys. Rev. Lett. 70, 3792 (1993).

[6] W. Cai, P. Hu, T. F. Zheng, B. Yudanin, and M. Lax, Phys. Rev. B 41, 3513 (1990).

[7] A. O. Caldeira and A. J. Leggett, Phys. Rev. Lett. 46, 211 (1981).

[8] J. L. Mateos and J. V. José, Physica A 257, 434 (1998).

[9] J. L. Mateos, Phys. Lett. A 256, 113 (1999).

[10] V. Berdichevsky and M. Gitterman, Phys. Rev. E 59, R9 (1999).
[11] M. Arrayás, I. Kh. Kaufman, D. G. Luchinsky, P. V. E. McClintock, and S. M. Soskin, Phys. Rev. Lett. 84, 2556 (2000).

[12] J. Iwaniszewski, Phys. Rev. E 68, 027105 (2003).

[13] J. Iwaniszewski, I. Kh. Kaufman, P. V. E. McClintock, and A. J. McKane, Phys. Rev. E 61, 1170 (2000).

[14] J. Iwaniszewski, Phys. Rev. E 54, 3173 (1996).

[15] M. V. Berry, Eur. J. Phys. 2, 91 (1981).

[16] G. Karner, J. Stat. Phys. 77, 867 (1994).

[17] K. Y. Tsang and K. L. Ngai, Phys. Rev. E 56, R17 (1997).

[18] A. Loskutov, A. B. Ryabov, and L. G. Akinshin, J. Phys. A 33, 7973 (2000).

[19] J.-P Eckmann, and D. Ruelle, Rev. Mod. Phys. 57, 617 (1985).

[20] J.-P Eckmann, S. O. Kamphorst, D. Ruelle, and S. Ciliberto, Phys. Rev. A 34, 4971 (1986).

[21] E. D. Leonel, and J. K. L. da Silva, Physica A 323, 181 (2003).

[22] G. A. Luna-Acosta, G. Orellana-Rivadeneyra, A. MendozaGalván, and C. Jung, Chaos, Solitons Fractals 12, 349 (2001).

[23] A.-L. Barabási and H. E. Stanley, Fractal Concepts in Surface Growth (Cambridge University Press, Cambridge, 1985). 